\title{
Neutral Clouds Within the Local X-ray Plasma
}

\author{
Jürgen Kerp and Jochen Pietz \\ Radioastronomisches Institut der Universität Bonn, D-53121 Bonn, Germany
}

\begin{abstract}
We present new results based on the analysis of ROSAT PSPC observations towards nearby neutral clouds. These clouds are detectable as deep soft $\mathrm{X}$-ray absorption features within the $\frac{1}{4} \mathrm{keV}$ energy band. Towards one of these clouds, optical absorption line measurements determine an upper distance limit of $D \leq 60 \mathrm{pc}$. The presented data suggest that this filament is only a part of a much more extended local-cloud structure. Here we demonstrate that we most probably detected a local neutral atomic hydrogen "wall" in the direction of a low-velocity arc (Pietz 1994).
\end{abstract}

\section{Basic information}

Our view is focused towards the galactic sky region centered at $l \sim 85^{\circ}$, $b \sim 37^{\circ}$ (Fig. 1). This area partly covers an arc-like high $\mathrm{H}$ I column density structure (Pietz 1994). Here the low-velocity gas reaches $\mathrm{H}$ I column densities of about $N_{\mathrm{HI}} \simeq 5 \cdot 10^{20} \mathrm{~cm}^{-2}$, and the $\mathrm{H} \mathrm{I} 21-\mathrm{cm}$ linewidth indicates kinetic cloud temperatures of $T_{\text {kin }} \simeq 100 \mathrm{~K}$.

Towards the nearby low-velocity cloud LVC $88+36-2$, part of the cloud structure of interest, Wennmacher et al. (1992) determined an upper distance limit of 60 pc. This suggests that this cloud may either be inside the Local Bubble, or form part of its boundary region. Kerp et al. (1993) analysed a pointed ROSAT PSPC observation towards LVC 88+36-2 and confirmed the suggestion that the cold, dense neutral hydrogen filament is embedded within the local X-ray plasma. They derived a three-dimensional structure of X-ray emitting and absorbing matter from the analysis of soft X-ray "colours". Because of the strong energy dependence of the photoelectric absorption cross section $\left(\sigma \propto E^{-3}\right)$, the quantitative comparison of different energy bands provides a means of disentangling the various components within the interstellar medium. The analysis of Kerp et al. (1993) suggests a three-component model of the soft X-ray radiation transfer equation (MODEL I):

$$
I_{\mathrm{obs}}=I_{\mathrm{foregr}}+I_{\mathrm{backgr}} \cdot e^{-\sigma \cdot N_{\mathrm{HI}}(\mathrm{LHB})}+I_{\mathrm{halo} / \mathrm{extra}} \cdot e^{-\sigma \cdot N_{\mathrm{HI}}(\text { total })}
$$

Here, LVC $86+38-2$ is embedded within the local X-ray plasma. A fraction of the local X-ray emission originates in front of the cloud $\left(I_{\text {foregr }}\right)$, while the residual local $\mathrm{X}$-ray radiation is produced from beyond the low-velocity cloud ( $I_{\text {backgr }}$ ). Moreover, the H I 21 -cm line data suggest that LVC $86+38-2$ is only a part of a much more extended H I structure. We analysed four pointed PSPC 
observations towards this galactic sky area to search for evidence in soft Xray colours that also these low-velocity clouds are probably located within the Local Bubble. We find that the above radiation transfer model provides a good quantitative fit to the observational data. This implies that also the very extended $\mathrm{H}$ I column density structure, which we called the "Basis" of the low-velocity arc, is embedded within the local X-ray plasma.

\section{Alternative Explanation and Limits of the X-ray Data Analysis}

We analysed the ROSAT PSPC data within the R1, R2, $1 / 4 \mathrm{keV}$ and $3 / 4 \mathrm{keV}$ energy bands. To improve the analysis we fitted the ROSAT PSPC R1:R2, $1 / 4 \mathrm{keV}: 3 / 4 \mathrm{keV}$ band ratios as well as the $1 / 4 \mathrm{keV}$ and $3 / 4 \mathrm{keV}$ band count rate plotted versus the total $N_{\mathrm{HI}}$ column density (scatter diagrams) simultaneously. This method allows us to constrain the intensity of the individual soft X-ray source terms of Eq. (1) as well as the temperature of the local and distant X-ray plasma. The parameters of the extragalactic background are fixed for the representation by a power-law of $E^{-1.5}$ (Almaini et al. 1996) and a $1 / 4 \mathrm{keV}$ intensity of $I_{\text {extra }}(1 / 4 \mathrm{keV})=2.3 \cdot 10^{-4} \mathrm{cts} \mathrm{s}^{-1} \operatorname{arcmin}^{-2}($ Barber et al. 1996).

By fitting the X-ray data in all 4 diagrams simultaneously (Fig. 2) we determined, in the $1 / 4 \mathrm{keV}$ band, for MODEL I (low-velocity clouds within the Local Bubble):

$$
\begin{aligned}
& T_{\mathrm{LHB}}=10^{5.8 \pm 0.1} \mathrm{~K} \text { and } T_{\text {halo }}=10^{6.2 \pm 0.1} \mathrm{~K}, \\
- & I_{\text {foregr }}=(3.5 \pm 0.5) \cdot 10^{-4} \mathrm{cts} \mathrm{s}^{-1} \operatorname{arcmin}^{-2} \text { and } I_{\text {backgr }}=(3.0 \pm 0.5) \\
& 10^{-4} \operatorname{cts~s}^{-1} \operatorname{arcmin}^{-2} \\
- & I_{\text {halo }}=(10 \pm 1.5) \cdot 10^{-4} \mathrm{cts} \mathrm{s}^{-1} \operatorname{arcmin}^{-2} .
\end{aligned}
$$

An alternative approach assumes that the low-velocity clouds are embedded within the warm neutral medium (WNM). In this case $I_{\text {backgr }}=0$ and the WNM forms the boundary of the local void of neutral matter (MODELII). Here, we found that the data are fitted best by a mixture of the hot halo plasma $T_{\text {hotter halo }} \simeq 10^{6.2} \mathrm{~K}$ (Pietz et al. and Wang, this volume) in addition to a cooler halo component with $T_{\text {cooler halo }} \simeq 10^{5.9} \mathrm{~K}$. For MODEL II we find:

- $T_{\mathrm{LHB}}=10^{5.8 \pm 0.1} \mathrm{~K}$, and a plasma mixture consisting of $T_{\text {cooler halo }}=$ $10^{5.9 \pm 0.1} \mathrm{~K}$ and $T_{\text {hotter halo }}=10^{6.2 \pm 0.1} \mathrm{~K}$,

$-I_{\text {local }}=(3.5 \pm 0.5) \cdot 10^{-4} \operatorname{cts~s}^{-1} \operatorname{arcmin}^{-2}$,

$-I_{\text {cooler halo }}=(30 \pm 5) \cdot 10^{-4} \mathrm{cts} \mathrm{s}^{-1} \operatorname{arcmin}^{-2}$ and $I_{\text {hotter halo }}=(6 \pm 1.5)$. $10^{-4} \mathrm{cts} \mathrm{s}^{-1} \operatorname{arcmin}^{-2}$.

This model of the X-ray emitting and absorbing matter also fits the X-ray data well and, if substantiated, would have the following important consequences: 
- The Local Bubble has an extent of less than $60 \mathrm{pc}$ in this direction

- The WNM with $N_{\mathrm{HI}}(\mathrm{WNM}) \simeq 1-3 \cdot 10^{20} \mathrm{~cm}^{-2}$ is located close to the low-velocity clouds $\left(D_{\mathrm{WNM}} \geq 60 \mathrm{pc}\right)$.

- The emission measure of the halo component in MODELII is 4 times higher than that of MODELI.

\section{Discussion}

Because of the limited energy resolution of the ROSAT PSPC the X-ray data do not allow to distinguish between the two models considered above. Therefore, supplementary data are necessary to find the most likely interpretation of the observed situation. Optical absorption line measurements will be ideal to serve for this supplementary information.

Wennmacher (1994) estimated the distance of the WNM towards the lowvelocity clouds distributed within the field of interest. He came to the conclusion that the bulk of the WNM has an average distance of about $D_{\mathrm{WMN}} \simeq$ $300 \mathrm{pc}$. This WNM structure has a neutral hydrogen column density ranging from $N_{\mathrm{HI}}=1$ to $3 \cdot 10^{20} \mathrm{~cm}^{-2}$. These values are close to the total neutral hydrogen column density observed in directions offset from the low-velocity clouds.

Quantitatively, this suggests that between the LVC $86+36-2\left(D_{\mathrm{LVC}} \simeq 60 \mathrm{pc}\right)$ and the WNM $\left(D_{\mathrm{WNM}} \simeq 300 \mathrm{pc}\right)$ there is only a weak increase of $N_{\mathrm{HI}}$. Hence, most likely the low-velocity clouds are really embedded within the local lowvolume density region. In this sense MODEL I is the most plausible solution. Therefore, we can suggest that also the "Basis" is located within the Local Bubble. Furthermore, the thin $\mathrm{H}$ I $21-\mathrm{cm}$ filaments, which are connecting the LVC 86+38-2 with the "Basis" in position and velocity, seem to be also local. Probably we found an $\mathrm{H}_{\mathrm{I}} 21-\mathrm{cm}$ wall within the Local Bubble located at a distance between $50-60 \mathrm{pc}$. This model has to be confirmed by further optical absorption line measurements towards the low-velocity arc and LVC 86+38-2.

\section{References}

Almaini O. et al. (1996): MNRAS 282, 295

Barber C.R., Roberts T.P., Warwick R.S. (1996): MNRAS 282, 157

Kerp J., Herbstmeier U., Mebold U. (1993): A\&A 268, L21

Pietz J. (1994): Diploma thesis, Universität Bonn

Wennmacher A., Lilienthal D., Herbstmeier U. (1992): A\&A 250, L150

Wennmacher A. (1994): Ph. D. Thesis, Universität Bonn 


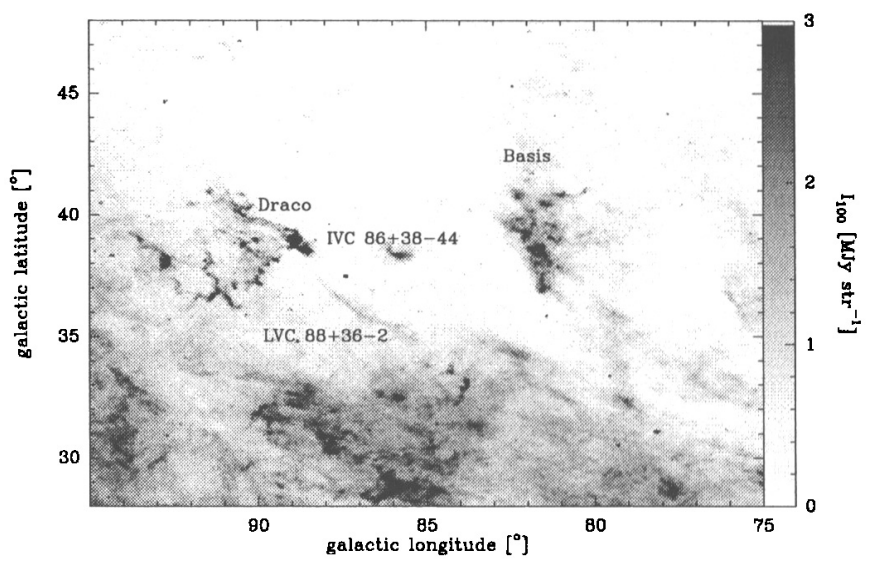

Fig. 1. IRAS $100 \mu \mathrm{m}$ map of the entire field of interest. The high-column-density clouds are located between $b=35^{\circ}$ and $42^{\circ}$. The newly investigated field is called the "Basis" and is visible centered at $l \simeq 82^{\circ}$ and $b \simeq 38^{\circ}$. From this area the low-velocity arc emerges to high galactic latitudes. The low-velocity cloud LVC $86+38-2$ is at a distance $D_{\text {LVC }} \leq 60 \mathrm{pc}$.
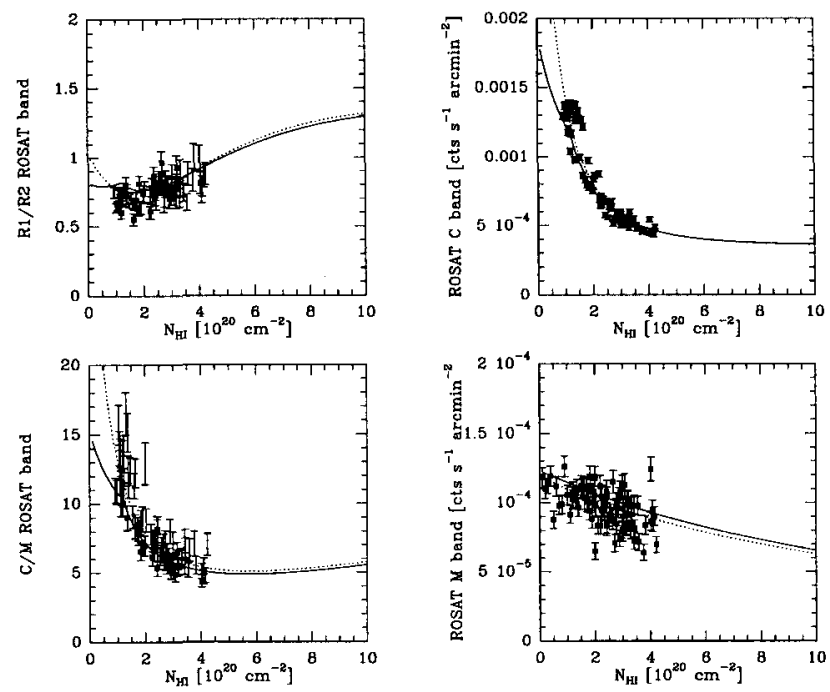

Fig. 2. Results of the analysis of four PSPC pointings towards the high-column-density filament centered at $l \sim 82^{\circ}, b \sim 37^{\circ}$ (Fig. 1). The X-ray data are simultaneously fitted for the $\mathrm{R} 1: \mathrm{R} 2,1 / 4 \mathrm{keV}: 3 / 4 \mathrm{keV}$ band ratios and the $1 / 4 \mathrm{keV}$ and $3 / 4 \mathrm{keV}$ scatterdiagrams. The solid lines represent the best-fit of MODEL I (low-velocity clouds within the Local Bubble) and the dotted lines the best-fit of MODEL II (low-velocity clouds outside the Local Bubble). 